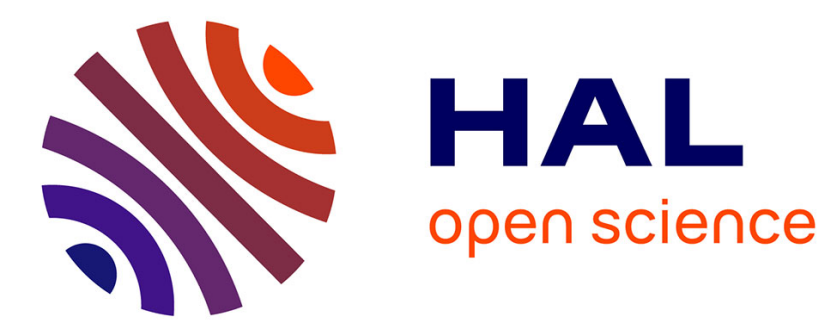

\title{
Pour une histoire des symbioses énergétiques et matérielles
}

\author{
Jean-Baptiste Fressoz
}

\section{To cite this version:}

Jean-Baptiste Fressoz. Pour une histoire des symbioses énergétiques et matérielles. Annales des mines - Série Responsabilité et environnement, 2021, pp.7-11. hal-03101307

\section{HAL Id: hal-03101307 https://hal.science/hal-03101307}

Submitted on 11 Jan 2021

HAL is a multi-disciplinary open access archive for the deposit and dissemination of scientific research documents, whether they are published or not. The documents may come from teaching and research institutions in France or abroad, or from public or private research centers.
L'archive ouverte pluridisciplinaire $\mathbf{H A L}$, est destinée au dépôt et à la diffusion de documents scientifiques de niveau recherche, publiés ou non, émanant des établissements d'enseignement et de recherche français ou étrangers, des laboratoires publics ou privés. 


\title{
Pour une histoire des symbioses énergétiques et matérielles
}

\author{
Par Jean-Baptiste FRESSOZ \\ Historien, Centre de recherches historiques CNRS-EHESS
}

\begin{abstract}
Avec l'urgence climatique, l'expression « transition énergétique » a acquis un tel prestige que les historiens en sont venus à l'employer pour décrire toutes sortes de processus, y compris ceux qui furent, à rigoureusement parler, des additions énergétiques. Le problème de la « transition énergétique » est qu'elle projette un passé qui n'existe pas sur un futur pour le moins fantomatique. Cet article propose une nouvelle façon d'aborder l'histoire de l'énergie en tant que dynamique d'accumulation symbiotique.
\end{abstract}

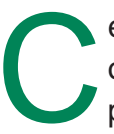
es dernières années ont vu paraître de nombreux ouvrages portant sur l'histoire de l'énergie. On peut se réjouir de ce renouveau d'intérêt, on peut aussi regretter que ces ouvrages se soient placés sous la bannière de la «transition ». Avec l'urgence climatique, ce mot a acquis un tel prestige, une telle centralité, que les historiens en sont venus à l'employer pour décrire toutes sortes de processus, y compris ceux qui furent, à rigoureusement parler, des additions énergétiques ${ }^{(1)}$.

La révolution industrielle est ainsi présentée comme une « transition » du bois vers le charbon, comme le passage d'une « économie organique » à une " économie minérale ». On peut lire dans un ouvrage de référence récent que le pétrole et l'électricité au $X X X^{\text {e }}$ siècle furent des « transitions énergétiques » - alors que l'électricité accroît la consommation de houille et que le pétrole ne la réduit pas ${ }^{(2)}$ forcément. La vision « phasiste » du monde matériel est si profondément ancrée que des historiens opposent un $\mathrm{XIX}^{\mathrm{e}}$ siècle du charbon à un $\mathrm{XX}$ e siècle du pétrole - et en tirent des conclu-

(1) PODOBNIK Bruce (2005), Global Energy Shifts. Fostering Sustainability in a Turbulent Age, Philadelphia, Temple University Press ; FOUQUET Roger (2008), Heat, Power and Light, Revolutions in Energy Services, Chetelnham, Edward Elgard; RHODES Richard (2018), Energy a Human History, New-York, Simon \& Schuster, 2018 ; GRUBLER Arnulf (2012), "Energy transitions research: Insights and Cautionary Tales", Energy Policy, vol. 50, pp. 8-16 ; WILSON Charlie \& GRUBLER Arnulf (2011), "Lessons From the History of Technological Change for Clean Energy Scenarios and Policies", Natural Resources Forum, vol. 35, pp. 165-184 ; DEBEIR Jean-Claude, DELÉAGE Jean-Paul \& HÉMERY Daniel (2013), Une histoire de l'énergie, Paris, Flammarion ; BOUVIER Yves \& LABORIE Léonard (2016), L'Europe en Transitions. Énergie, mobilité, communication, $X V I^{1}{ }^{e}-\left.X X\right|^{e}$ siècles, Paris, Nouveau Monde éditions : MASSARD-GUILBAUD Geneviève \& MATHIS Charles-François (2019), Sous le soleil. Systèmes et transitions énergétiques du Moyen Âge à nos jours, Paris, Presses de la Sorbonne. Un petit contrepoint : FRESSOZ Jean-Baptiste (2013), "Pour une histoire désorientée de l'énergie ", Entropia, vol. 15. (2) KANDER Astrid, MALAMINA Paolo \& WARDE Paul (2013), Power to the people. Energy in Europe Over the Last Five Centuries, Princeton, Princeton University Press, p. 251. sions hasardeuses sur l'histoire et la nature du pouvoir ${ }^{(3)}$. La prodigieuse lenteur de l'actuelle « transition énergétique » n'a pas non plus annulé les présomptions sur celles qui sont supposées avoir eu lieu par le passé (4).

Le problème de ces travaux n'est pas tant leur base empirique que ce qu'ils choisissent d'étudier. En se focalisant sur les transitions, l'histoire de l'énergie oriente les « leçons » que l'on infère du passé ${ }^{(5)}$. Cet article propose d'aborder la question par un autre angle : non pas celui des dynamiques, ni même celui des persistances, mais en considérant les relations symbiotiques qui se nouent entre énergies et matières ${ }^{(6)}$.

(3) MITCHELL Tim (2011), Carbon Democracy. Political Power in the Age of Oil, Londres, Verso.

(4) SMIL Vaclav (2010), Energy Transitions. History, Requirements, Prospects, Santa Barbara, Praeger.

(5) Certains auteurs en font mention en passant le fait que les énergies s'additionnent plus qu'elles ne se substituent, tout en renforçant le caractère transitionniste de l'histoire. C'est qu'il ne faudrait pas gâcher le message optimiste et motivationnel très prisé par d'innombrables rapports sur la transition. Un exemple frappant : SOVACOOL Benjamin (2016), "How long will it take? Conceptualizing the temporal dynamics of energy transitions", Energy Research \& Social Science, vol. 13, pp. 202-215.

(6) L'étude des persistances est extrêmement importante et ce sont ces travaux qui nourrissent cet article. Pour la sidérurgie au charbon de bois, voir SCHALLENBERG Richard H., "Evolution, adaptation and survival: the very slow death of the American charcoal iron industry", Annals of Science, vol. 32, n4, pp. 341-358. Sur l'hydraulique: HUNTER Louis C. (1979), History of Industrial Power in the United States, 1750-1930, vol. I, Waterpower in the Century of Steam, Charlottesville, University Press of Virginia, et BENOÎT Serge, D'eau et de feu : forges et énergie hydraulique, XVIIle-XXe siècles. Une histoire singulière de l'industrialisation française, Rennes, Presses Universitaires de Rennes, 2020. Sur la force animale : TARR J. A. (2007), The Horse in the City. Living Machines in the Nineteenth Century, Baltimore, The John Hopkins University Press ; JARRIGE François \& KASDI Mohamed, "Moteurs animés des filatures ", in JARRIGE François \& VRIGNON Alexis (2020), Face à la puissance. Une histoire des énergies alternatives à l'âge industriel, Paris, La Découverte. Sur les persistances technologiques en général, voir le livre très percutant de EDGERTON David (2012), Quoi de neuf? Une histoire globale des techniques au XXe siècle, Le Seuil. 


\section{Symbioses industrielles}

Commençons par l'exemple, canonique entre tous, de la révolution industrielle. Depuis les années 1980, les historiens de l'énergie ont ressuscité cette notion en la réinterprétant comme la transition séculaire d'une « économie organique » (l'expression remonte à Werner Sombart) reposant sur le bois, la force musculaire et l'hydraulique, vers une « économie minérale » ou un « capitalisme fossile » fondé sur le charbon, cette « forêt souterraine » qui permit à l'Europe d'échapper à la « contrainte photosynthétique » (7).

Cette interprétation repose sur des calculs énergétiques qui tendent à minorer le rôle des énergies dites traditionnelles et qui accentuent la révolution produite par le charbon ${ }^{(8)}$. Derrière la froide objectivité des courbes énergétiques ascendantes du $\mathrm{XVI}^{\mathrm{e}}$ au $\mathrm{XIX}^{\mathrm{e}}$ siècles se cachent des choix discutables sur ce qui entre dans le calcul. Par exemple, selon les données collectées par Paul Warde - qui sont à l'arrière-plan des analyses d'Anthony Wrigley ou de Vaclav Smil sur la révolution industrielle -, le bois ne jouerait plus aucun rôle dans le mix énergétique britannique au milieu du XIXe siècle ${ }^{(9)}$. Pourtant les mines de charbon anglaises engloutissent d'énormes quantités de bois : des poutres, des étais, des perches et des planches destinées à boiser les galeries - en tout, 4,5 millions de tonnes en $1913^{(10)}$. Cela signifie qu'en 1913, la Grande-Bretagne utilisait beaucoup plus de bois pour extraire son charbon qu'elle n'en brûlait au milieu du XVIII siècle. Malgré des efforts d'économie, réalisés au mépris de la sécurité des mineurs, la consommation de bois d'œuvre demeure proportionnelle à l'extraction du charbon (en France, de l'ordre de 3-4 \% du charbon extrait) loin dans le $X X^{e}$ siècle ${ }^{(11)}$. Cet exemple témoigne d'un phénomène général : bien plus qu'une transition du bois vers le charbon ou des matières organiques vers les matières minérales, l'industrialisation fut avant tout une mise en relation symbiotique des trois règnes.

Cette relation était une évidence pour tous les forestiers de l'époque : l'un d'eux se gausse des « jugements superficiels », de ceux qui " se figurent que grâce à l'em-

(7) SIEFERLE Rudolf (2001), The Subterranean Forest: Energy Systems and the Industrial Revolution, Cambridge, The White Horse Press ; WRIGLEY Anthony (2010), Energy and the English industrial revolution, Cambridge, Cambridge University Press.

(8) Cela tient à plusieurs raisons : 1) l'approche est centrée sur la production énergétique et non sur les services énergétiques effectivement rendus ; 2) faute de données, il est difficile de bien mesurer certaines énergies organiques ; 3) les paramètres retenus pour l'efficacité des convertisseurs (moulin hydraulique vs. machine à vapeur, par exemple) accroissent l'impact du charbon. Sur ce point, voir FRESSOZ Jean-Baptiste, « Pour une histoire matérielle de la lumière ", in JARRIGE F. \& VRIGNON A., Face à la puissance, op. cit.

(9) WARDE P. (2007), Energy Consumption In England and Wales, Consiglio Nazionale delle Ricerche Istituto di Studi sulle Società del Mediterraneo, p. 69 et appendix 2 - Wrigley, Energy and the Industrial Revolution, op. cit., p. 37 ; SMIL Vaclav, Energy transition..., op. cit., p. 79.

(10) REDMAYNE Richard (1923), The British Coal Industry During the War, Oxford, Clarendon Press, p. 44. Selon Paul Warde, 3,6 millions de $\mathrm{m}^{3}$ correspondent à la fourchette haute du pic du bois de feu au milieu du XVIII siècle en Angleterre et au Pays de Galles. Voir WARDE P., op. cit., p. 38.

(11) COULON Jean-Pierre (1940), Les bois de mine, Paris, Presses universitaire de France. ploi du fer, de l'acier et du charbon, le bois est un produit de plus en plus délaissé ${ }^{(12)}$ ». Pendant tout le XIX $\mathrm{X}^{\mathrm{e}}$ siècle, l'utilisation du bois de feu résiste bien face au charbon. $\mathrm{Au}$ début du $X X^{e}$ siècle, les forestiers Zon et Sparhawk estiment qu'en Amérique du Nord comme en Europe, près de la moitié du bois est abattu pour être brûlé ou carbonisé ce serait $80 \%$ sur les autres continents ${ }^{(13)}$. Aux États-Unis, la sidérurgie au bois continue de croître jusque dans les années 1890, son déclin date seulement de l'entre-deux guerres $^{(14)}$. La France brûlait 20 millions de $\mathrm{m}^{3}$ de bois en 1876 et encore 17 millions en $1908^{(15)}$. La baisse du bois de feu concerne surtout les villes. À Paris, elle est compensée par la montée de la houille, bien sûr, mais aussi par celle du charbon de bois, dont la consommation par habitant croît jusque dans le dernier quart du $\mathrm{XIX}^{\mathrm{e}}$ siècle ${ }^{(16)}$.

À cette date, la chimie prend le relais : les forêts de l'Yonne et du Nivernais qui approvisionnaient Paris sont dorénavant exploitées sur place grâce à l'industrie de la pyrolyse du bois qui à la fin des années 1880 s'implante aux points névralgiques du flottage. Les méthodes modernes de carbonisation (la cornue Lambiotte) permettent d'obtenir de meilleurs rendements, donc davantage de charbon et surtout de récupérer des produits chimiques à forte valeur ajoutée qui incitent à produire davantage de charbon. La fin du flottage sur l'Yonne dans les années 1920 n'est donc pas le signe d'un déclin du bois-énergie, mais plutôt celui de sa reconfiguration par la chimie industrielle ${ }^{(17)}$.

Même si le bois de feu recule dans certains pays ou certaines industries, ce recul est largement compensé par l'augmentation de la consommation de bois d'œuvre. La consommation britannique de bois d'œuvre est ainsi multipliée par 6 entre 1830 et 1930, et par 3 rapportée au nombre d'habitants ${ }^{(18)}$. De tous les pays européens, c'est la Grande-Bretagne, championne du charbon, qui importe aussi le plus de bois : 12 millions de $\mathrm{m}^{3}$ à la fin du XIXe siècle, soit deux fois et demi la production des forêts françaises. Grâce au progrès des transports, elle peut s'approvisionner en quantité croissante à un prix décroissant ( $35 \%$ dans le dernier quart du XIXe siècle). D'autres pays européens mieux dotés en forêts suivent aussi cette tendance : la Belgique voit ses importations multipliées par

(12) MÉLARD A. (1900), Insuffisance du bois d'œuvre dans le monde, Paris, Imprimerie Nationale, p. 3.

(13) STAMP Dudley (1928), "The Forest of Europe: Present and Future", Empire Forestry Journal, vol. 7, n², pp. 185-202.

(14) SCHALLENBERG, art. cité.

(15) Voir Statistique forestière, Paris, Imprimerie nationale, 1878, p. 108 ; et DAUBRÉE Lucien (1912), Statistique et Atlas des forêts de France, Paris, Imprimerie nationale, vol. 2, p. 334.

(16) La consommation de charbon de bois de Paris passe de $100000 \mathrm{~m}^{3}$ en 1800 à $500000 \mathrm{~m}^{3}$ en 1880. BECQUEREL Antoine César M. (1866), « Mémoire sur les forêts et leur influence climatérique ", Mémoire de l'Académie des sciences, vol. 35, pp. 5085010 ; et DE LA TOUR Imbart (1900), La crise agricole en France et à l'étranger, thèse de droit, Paris, p. 103.

(17) BRAQUE R., "Les industries de la carbonisation du bois en France ", L'Information Géographique, 1949, vol. 13, n¹, pp. 2833.

(18) HILEY W. E. (1930), The Economics of Forestry, Oxford, Oxford University Press, p. 38. IRIARTE-GONI Iñaki \& AYUDA Maria-Isabel (2012), "Not only subterranean forests: Wood consumption and economic development in Britain (1850-1938)", Ecological Economics, vol. 77, pp. 176-184. 
6 entre 1860 et 1900 , et l'Allemagne, malgré ses forêts résineuses de plaine à fort rendement, double les siennes entre 1888 et 1898. La France qui dispose surtout de forêts de taillis pour le bois à brûler est obligée à la fin du $\mathrm{XIX}$ siècle d'importer 3 millions de $\mathrm{m}^{3}$ de bois d'œuvre, soit la moitié de sa production nationale ${ }^{(19)}$.

Que fait-on de tout ce bois d'œuvre ? Avec des variations nationales, la construction arrive en tête (environ la moitié de la demande), vient ensuite la production de papier (un cinquième), les transports, puis l'emballage (tonneaux et caisses) ${ }^{(20)}$. D'autres usages massifs sont tombés dans l'oubli : au début du XX $X X^{\text {e }}$ siècle, la plupart des grandes artères londoniennes sont recouvertes de pavés de bois (un matériau glissant, mais apprécié pour la douceur de son roulement) ${ }^{(21)}$, comme l'est un cinquième des chaussées parisiennes $^{(22)}$.

Les techniques emblématiques de la « révolution industrielle » reposent sur le bois. Les «chemins de fer » auraient tout aussi bien pu s'appeler « chemins de bois » : dans les années 1890, la maintenance du réseau américain requiert 73 millions de traverses par an, soit 14 millions de $\mathrm{m}^{3}$ de grumes, une production équivalant à 20 millions d'hectares de forêt. Un dixième de la production forestière des États-Unis serait ainsi consacré au train ${ }^{(23)}$. Au même moment, la consommation en fer pour les rails était de 1,5 millions de tonnes par an, un poids à peu près équivalent aux traverses susmentionnées ${ }^{(24)}$. À cela, il faut ajouter que les fabricants de locomotives préféraient recourir à des aciers « de luxe » issus de la sidérurgie au bois (au moins pour les chaudières et les essieux), ceux-ci étant moins cassants que l'acier au coke.

La construction repose aussi sur une alliance du bois et du charbon : la cuisson des briques est un gouffre énergétique - le troisième consommateur industriel de charbon américain - et le premier poste de consommation de bois d'œuvre ${ }^{(25)}$. De manière plus anecdotique, le fameux Palais de cristal de l'Exposition universelle de Londres en 1851, symbole de la modernité matérielle (fer/ verre) du $\mathrm{XIX}^{\mathrm{e}}$ siècle, comportait au moins trois fois plus de bois que de fonte. L'une des vraies innovations liées à ce bâtiment - inspiré des serres agricoles - réside dans la production mécanique de pièces en bois standardisées ${ }^{(26)}$.

(19) MÉLARD, op. cit ; DAHEUR Jawad (2017), " La sylviculture allemande et ses hectares fantômes au tournant des XIXe et XXe siècles ", Revue Forestière Française, vol. 69, n³, pp. 227-239.

(20) GLESINGER Egon (1932), Le Bois en Europe, Paris, Sirey.

(21) On le voit sur la Bartholomew's road surface map of London \& Neighbourhood, https://vu.contentdm.oclc.org/digital/collection/krt/ id/1618/rec/1 (consulté le 21 octobre 2020).

(22) BEAUVERIE J. (1905), Le Bois, Paris, Gauthier-Villar, vol. 2, p. 1327. GUILLERME André (1995), Bâtir la ville : révolutions industrielles dans les matériaux de construction, Paris, Champs Vallon, pp. 220-222.

(23) TRATMAN \& FERNOW (1891), " Consommation des traverses par les chemins de fer des États-Unis d'Amérique ", Revue générale des chemins de fer, vol. 14, pp. 44-49.

(24) Monthly Summary of Commerce and Finance of the United States, Washington, Government Printing Office, Juillet 1900, p. 258.

(25) LE CHATELIER Henri (1925), Le Chauffage industriel, p. 454 ; "How much coal is burned in clay products plants", The Black Diamond, 1919, vol. 63, n¹, p. 321.

(26) Report of the Commissioners for the exhibition of 1851, Londres, Clowes, 1852, p. 69.
Certaines innovations industrielles eurent aussi un rôle essentiel dans l'augmentation de la production de bois. On pense naturellement aux technologies d'attaques - les chemins de fer qui ouvrent de nouveaux fronts à l'exploitation forestière ou les scieries à vapeur -, mais dans ce domaine la vraie révolution aura lieu plus tard, après la Seconde Guerre mondiale avec la diffusion de la tronçonneuse et des engins forestiers ${ }^{(27)}$. La grande innovation du $X X^{e}$ siècle, qui accroît indirectement la production de bois, c'est le papier de cellulose. Au début du XXe siècle, dans les pays riches, le papier est le troisième poste de consommation de bois après l'énergie et la construction ${ }^{(28)}$. Il s'agit d'une alliance à part égale de bois et de charbon : chaque tonne de papier nécessitant environ une tonne et demie de chacune de ces matières (en plus de produits chimiques) ${ }^{(29)}$. Son intérêt est de valoriser les immenses forêts de résineux du Canada et des pays scandinaves, d'utiliser des bois plus jeunes et donc de permettre des rotations forestières plus rapides : à travers l'industrie papetière, le charbon accroît donc la production de bois ${ }^{\left({ }^{30}\right)}$.

\section{Carbon fallacy}

« Vous pourriez facilement traverser tout le nord de l'Angleterre », écrivait George Orwell en 1937, « sans vous rendre compte que, cent mètres au-dessous de la route, des mineurs sont en train de tailler du charbon ». « Pourtant, ajoutait-il, ce sont eux qui font avancer la voiture ${ }^{(31)} »$. Pressés de raconter l'épopée du pétrole et de l'électricité, les historiens filent aussi à toute vitesse vers le futur : passé le cap des années 1900, le charbon passe à l'arrière-plan, analysé en tant que « persistance de l'ancien (32) », alors même que sa plus forte croissance a eu lieu dans les années 2000.

Si le pétrole s'ajoute au charbon plus qu'il ne le remplace, c'est parce que les deux matières n'entrent que marginalement en concurrence. Les spécialistes des années 1930 insistent au contraire sur leur complémentarité ou même sur leur « solidarité ${ }^{(33)}$ ». Le pétrole permet surtout de nouveaux usages et, au premier chef, de faire rouler des camions et des voitures. Or, l'automobile, par ses consommations induites, consomme davantage de charbon que de pétrole. En 1934, l'ingénieur en chef de l'Anglo-Iranian Company calcule que l'industrie automobile, la construction des pétroliers, des réservoirs et des raffineries britanniques ont requis 13 Mt d'acier de 1918 à 1934 ,

(27) JOSEPHSON Paul (2002), Industrialized Nature, Brute Force Technology and the Transformation of the Natural World, chapitre 2. (28) GLESINGER, op. cit., p. 315.

(29) Dans l'entre-deux guerres, l'industrie papetière anglaise brûle 2,5 millions de tonnes de charbon légèrement plus que l'industrie textile. BRADLEY John R. (1935), Fuel and Power in the British Empire, Washington, Government Printing Office, p. 52.

(30) HILEY W. E., op. cit., p. 10.

(31) ORWELL George (1937), The Road to Wigan Pier, New-York, Harcourt, 1958, p. 34.

(32) Power to the People, op. cit., p. 258

(33) BRUNSCHWIG Robert (1933), "Charbon et pétrole dans l'économie moderne ", Annales de l'Office national des combustibles liquides, vol. $8, n^{\circ} 2$, p. 266. Hormis dans le transport maritime, où il y a effectivement une substitution grâce au rendement supérieur des moteurs diesel par rapport aux machines à vapeur. 
et donc 53 Mt de charbon. La Grande-Bretagne n'ayant consommé que $21 \mathrm{Mt}$ de pétrole sur la même période, il en concluait que chaque tonne de pétrole avait nécessité 2,5 tonnes de charbon (34). Louis Pineau, le directeur de l'Office français des combustibles liquides, arrive à un résultat comparable : la production d'une voiture en France nécessitait à peu près autant de charbon (7 tonnes) qu'elle ne consommait d'essence sur la totalité de son cycle d'usage ${ }^{(35)}$. Conclusion : le pétrole « épaule le charbon ». Ces calculs sont des estimations basses, puisqu'il faudrait y ajouter le charbon employé à l'adaptation du réseau routier ${ }^{(36)}$ ou encore celui nécessaire au raffinage et au transport du pétrole (37).

En fait, Orwell ne croyait pas si bien dire : avant la Seconde Guerre mondiale, le charbon a effectivement fait rouler de nombreuses voitures, car on en tirait du benzol, un carburant qui améliorait les qualités anti détonantes de l'essence. Dans les années 1900, en France, la plupart des autobus et la majorité des automobiles roulent non pas à l'essence de pétrole, mais au benzol. Selon un journaliste de l'époque, « c'est le benzol qui a développé l'automobile ${ }^{(38)}$ ». Pendant la Première Guerre mondiale, les États belligérants obligent les cokeries et les usines à gaz à récupérer le benzol qui est aussi un composant des explosifs. La production s'accroît fortement dans l'entredeux guerres en lien avec la perspective d'un conflit militaire. En France, le gouvernement encourage l'utilisation d'un mélange de benzol et d'alcool dénommé le « carburant national ». Dans les grands pays charbonniers, le benzol joue un rôle non négligeable : en Angleterre, il représente un dixième de l'essence et même jusqu'à un quart en Allemagne ${ }^{(39)}$.

Pendant la Guerre froide, le pétrole ne joue qu'un rôle secondaire en tant qu'énergie industrielle, la période correspondant plutôt à l'apogée du charbon qui se modernise sous la houlette d'États dirigistes : l'Angleterre connaît son pic de consommation en 1956, le charbon représenterait alors $95 \%$ de son énergie primaire ${ }^{(40)}$; en France, le pic de consommation est atteint en 1962, avec

(34) DALLEY (1935), "Oil as an ally of coal", in BRADLEY John R., Fuel and Power in the British Empire, US department of Commerce. (35) PINEAU Louis, "Le Pétrole ", Le Génie civil, vol. 106, n¹4, pp. 338-340.

(36) L'explosion de la production de ciment est contemporaine de I'automobile : la production mondiale passe de $2 \mathrm{Mt}$ en 1880 à 235 Mt en 1956. Chaque tonne de ciment nécessite alors 300 kilos de charbon. Avant 1960, le ciment est produit quasi exclusivement avec du charbon. La part du ciment dévolue aux routes est variable selon les pays : 10 \% en France, $25 \%$ aux États-Unis, dans les années 1950. LACOSTE Yves (1957), "L'industrie du ciment », Annales de Géographie, n³57, pp. 411-435. Sur le ciment, voir la thèse en cours de Nelo Magalhaes.

(37) Les trois quarts des tankers sont encore des steamers en 1929. Voir SCHUMAN Jacques (1936), Le transport maritime des pétroles, Paris, Pedone, p. 54. Les raffineries américaines consomment 3 millions de tonnes de charbon par an dans les années 1920.

(38) Bulletin municipal officiel de la ville de Paris, 5 décembre 1911, p. 4299

(39) BIHOREAU Charles (1927), « Le benzol et son emploi comme carburant ", Annales de l'Office national des carburants liquides, pp. 281-308.

(40) WARDE P., 2007, p. 69.
75 millions de tonnes ${ }^{(41)}$. L'électrification renforce encore la centralité du charbon : des centrales thermiques sont établies directement dans les bassins houillers, les plus grandes pouvant engloutir 4 millions de tonnes de charbon par an ${ }^{(42)}$. La politique suivie dans les pays capitalistes n'est pas de favoriser le pétrole, mais plutôt de gérer au mieux sa coexistence avec le charbon : au premier, l'essence, les lubrifiants, les plastiques, à savoir des produits plus rémunérateurs ; au second, la production électrique, le ciment et la sidérurgie. Les progrès dans le raffinage permettent justement de réduire la part des fractions lourdes destinées à l'industrie. La consommation du fuel industriel ne décolle en Europe qu'à partir de 1958 avec l'afflux de pétrole moyen-oriental bon marché et la diffusion véritablement massive de l'automobile qui rendent le pétrole compétitif par rapport au charbon ${ }^{(43)}$.

Contrairement à l'hypothèse de Tim Mitchell, ce n'est donc pas une transition inexistante vers le pétrole qui a pu dompter les mineurs. En France, la grande grève de 19471948 dans les charbonnages du Nord fut vaincue grâce à la répression militaire, aux licenciements, et surtout, grâce au charbon américain et allemand ${ }^{(44)}$. Pour briser la grève, le gouvernement français importe jusqu'à un million de tonnes de charbon des États-Unis par mois grâce au plan Marshall. Celui-ci, parfois présenté comme le cheval de Troie du pétrole en Europe de l'Ouest ${ }^{(45)}$, a davantage contribué à la modernisation des mines. En France, après EDF, c'est Charbonnage de France qui reçoit le plus d'argent du plan Marshall (46).

Le charbon est au cœur de la stratégie des États-Unis en Europe ${ }^{(47)}$. C'est sous leur égide qu'est lancé le grand plan « charbon pour l'Europe ». Son but : reconstruire et intégrer l'industrie européenne grâce au charbon de la Ruhr, tout en ménageant des débouchés à leurs propres houillères. L'administration du plan Marshall considère d'un mauvais œil la politique pétrolière expansionniste de la France qui cherche à renforcer sa souveraineté énergétique. Ce plan - qui deviendra celui de Schuman en 1950, puis la CECA

(41) Les pics d'extraction ont lieu avant : 1913 en Grande-Bretagne, et 1958 en France.

(42) PRÊCHEUR C. (1961), « L'électricité en France en 1959 et 1960 », L'Information géographique, vol. 25, n³, pp. 109-120 ; et CHALINE C. (1962), "Tendances actuelles de la production de charbon en Grande-Bretagne ", L'Information géographique, vol. 26, n4, pp. 169-171.

(43) BRUNET Roger (1961), « Le pétrole en Grande-Bretagne », L'Information géographique, vol. 25, n², pp. 69-77.

(44) FONTAINE Marion \& VIGNA Xavier (2014), " La grève des mineurs de l'automne 1948 en France », Vingtième siècle, n¹21, pp. 21-34.

(45) PAINTER David (2009), "The Marshall Plan and oil", Cold War History, vol. 9, n², pp. 159-175.

(46) CHARDONNET Jean (1951), " Le problème du charbon ", La revue économique, vol. 3, pp. 315-325 ; Rapport sur les aspects économiques du plan de modernisation des houillères, Paris, Lahure, 1949, pp. 7-9.

(47) HOLTER Darryl (1985), " Politique charbonnière et Guerre froide 1945-1950 », Le mouvement social, n¹30, pp. 33-53, et PERRON Régine (1996), Le marché du charbon, un enjeu entre l'Europe etles États-Unis de 1945à 1958, Paris, Éditions de la Sorbonne; MAMEHARA Keisuke (2016), « Du plan Monnet au plan Béthancourt. Comment ont évolué la politique charbonnière et la politique énergétique pendant les Trente glorieuses », thèse de l'Université de Paris IV. 
en 1954 - permet de protéger le gouvernement français des mineurs cégétistes du Nord, puisqu'il est dorénavant possible de puiser dans le charbon allemand. Enfin, le déclin du charbon en France après le plan Jeanneney de 1962 est beaucoup plus spectaculaire en valeur relative (du fait de l'afflux du pétrole moyen-oriental bon marché) qu'en valeur absolue : la France en consomme encore entre 40 et 50 millions de tonnes dans les années 19701980, c'est-à-dire deux fois plus qu'un siècle auparavant.

La vague de globalisation et la révolution néolibérale des années 1980 se sont aussi parfaitement accommodées du charbon. Aux États-Unis, l'élection de Reagan marque le début d'une énorme croissance charbonnière, lancée par les chocs pétroliers et qui aboutira en 2008 au pic historique de la consommation américaine à 1,2 Gt/an. Bien plus que le pétrole, c'est l'évolution technologique du charbon qui a transformé la culture politique des mineurs. L'extraction aux États-Unis migre des mines de Pennsylvanie vers le Wyoming, des bastions ouvriers socialistes au Midwest agricole et républicain. L'activité minière se transforme : plutôt que d'extraire le charbon du sous-sol, d'immenses engins enlèvent le sol qui se trouve au-dessus de lui ${ }^{(48)}$. Résultat : la productivité des mines triple aux États-Unis entre 1980 et 1995. L'ouvrier des mines à ciel ouvert du Wyoming extrait 20 tonnes de charbon par jour contre 1,5 tonnes pour les charbonnages de France dans les années $1950^{(49)}$. De même, en Allemagne, ce n'est pas le pétrole qui remplace le charbon, mais la lignite exploitée à ciel ouvert. Le charbon a aussi alimenté le rattrapage économique de la Chine, lui-même accéléré par la globalisation néolibérale. La consommation chinoise passe de 1,5 Gt à 4 Gt entre 2000 et 2015. Chaque année, la Chine brûle à peu près autant de charbon que la France dans toute son histoire. Le charbon n'est pas une énergie qui serait « moins moderne » que le pétrole. II est tout autant l'énergie de la « révolution industrielle » que celle d'Internet, qui n'est au fond qu'un réseau supplémentaire d'électrons.

Notons pour finir que si l'énergie focalise l'attention, son histoire n'est absolument pas exceptionnelle : au cours des deux derniers siècles, l'éventail des matières premières utilisées s'élargit sans cesse, et chacune des matières est consommée en quantités croissantes. Les matières premières ne deviennent jamais obsolètes et les processus de substitution sont largement compensés par les effets rebonds ou les réorientations d'usage. Entre 1900 et 2015, le poids total des matières premières consommées par l'économie mondiale a été multiplié par $12^{(50)}$. Depuis la Seconde Guerre mondiale, malgré la prolifération des produits de synthèse (plastiques, etc.), aucune grande matière première n'a décru, hormis la laine de mouton qui

(48) ANGLIER Jean-Pierre (1981), « Le charbon, industrie nouvelle ", Revue d'économie industrielle, vol. 16, pp. 1-15; GOODELL Jeff (2006), Big Coal: The Dirty Secret Behind America's Energy Future, New York, Houghton Mifflin.

(49) KUBY M. \& XIE Z. (2001), "The effect of restructuring on US Coal Mining labour productivity", Energy, vol. 26, n¹1, pp. 10151030.

(50) KRAUSMAN et al. (2018) "From resource extraction to outflows 1900-2015", Global environmental change. recule face aux fibres synthétiques, ce qui n'est d'ailleurs pas une bonne nouvelle pour l'environnement. Entre 1960 et 2010, sur les soixante-neuf principales matières premières, seules six ont vu leur consommation mondiale décliner. Et pour cinq d'entre elles, cette décroissance est due à leur toxicité et à des interdictions prononcées au plan national (51). Malgré la crise environnementale et la crise financière de 2008 , la consommation matérielle mondiale s'accélère : elle a cru de $53 \%$ entre 2002 et 2015. Entre ces deux dates, on a extrait $1000 \mathrm{Gt}$ de matières du sol, soit un tiers de tout ce qui avait été extrait depuis 1900.

\section{Conclusion}

Dans les années 1980, au moment où une nouvelle vague de charbon déferlait sur le monde, la transition devint la grande préoccupation des historiens de l'énergie. II faut voir dans ce paradoxe l'influence de la prospective énergétique très en vogue depuis les années 1970. Le discours de la transition émerge dans le milieu des futurologues qui pensent l'avenir énergétique des ÉtatsUnis après les chocs pétroliers. Si cette notion n'est pas un bon descripteur du passé, c'est que ce n'était tout simplement pas son but initial ${ }^{(52)}$. Et si elle a séduit les historiens, c'est parce qu'elle leur donnait aussi une certaine importance : en parlant la langue des technocrates, des pans entiers de l'historiographie (l'histoire économique, l'histoire des techniques, la révolution industrielle) semblaient soudainement acquérir une grande pertinence pour penser les défis du futur. Connaissant les ressorts des transitions énergétiques passées, les historiens pourraient ainsi devenir des experts de la transition à venir.

Le problème est que cette notion ne rendait pas du tout compte de la nature cumulative et symbiotique du passé énergétique et matériel. Elle permettait par contre d'imaginer une économie décarbonnée comme la suite, voire l'aboutissement d'un majestueux processus historique amorcé il y a deux siècles. Le problème de la « transition énergétique » est qu'elle projette un passé qui n'existe pas sur un futur qui reste fantomatique.

(51) II s'agit de l'amiante, du mercure, du beryllium, du tellurium et du thallium. Voir MAGEE Christopher L. \& DEVEZAS Tessaleno C. (2017), "A simple extension of dematerialization theory: Incorporation of technical progress and the rebound effect", Technological Forecasting \& Social Change, vol. 117, pp. 196-205.

(52) Sur ce point, voir FRESSOZ Jean-Baptiste, "Transition énergétique : essai de généalogie ", à paraître dans la revue Terrestres. 